\title{
Controlling Mechanical Properties of NiTi Scaffolds built by Selective Laser Melting
}

\author{
T. Bormann ${ }^{1,2}$, R. Schumacher ${ }^{1}$, B. Müller ${ }^{2}$, and M. de Wild ${ }^{1}$ \\ ${ }^{1}$ University of Applied Sciences Northwestern Switzerland, IMA, Muttenz, Switzerland. \\ ${ }^{2}$ University of Basel, Biomaterials Science Center, Basel, Switzerland.
}

\section{Introduction}

NiTi, a biocompatible and FDA-approved material, is a promising candidate for load-bearing implants as it combines low stiffness with high strength and exhibits properties like the pseudoelasticity, the shape memory effect and high damping capacities [1]. Selective laser melting (SLM) allows the fabrication of complex shaped constructs like open porous parts or filigree lattice structures with struts as thin as $200 \mu \mathrm{m}$ [2]. Using SLM for the fabrication of complexshaped NiTi-scaffolds, we aim to develop bone implants with an improved ossseoinductive performance owing to mechanical stimulation of surrounding tissues.

\section{Methods}

NiTi scaffolds and tensile test specimens were produced from pre-alloyed NiTi powder by SLM. For the fabrication of lattice structures, laser power and scanning velocity were varied from 60 to $95 \mathrm{~W}$ and 122 to $171 \mathrm{~mm} / \mathrm{s}$, respectively, resulting in an overall energy input of 60, 70, 100 and $130 \mathrm{~J} / \mathrm{mm}^{3}$ [3]. Mechanical testing was performed at room temperature using a universal testing machine.

\section{Results}

The compressive strength in the NiTi-scaffolds increases from $450 \mathrm{~N}$ to $1600 \mathrm{~N}$ and the elastic modulus from $110 \mathrm{MPa}$ to $660 \mathrm{MPa}$ in dependence of the applied energy density. The reason for the altered mechanical properties is an increase in the strut diameter and specimen mass caused by laser paths with expanded widths at higher energy densities [4]. Load-relieve tensile tests show the characteristic pseudoelastic behaviour in an SLM built tensile test specimen. We detected a complete shape recovery of maximal $3.4 \%$ strain.

\section{Conclusion}

The mechanical properties of lattice structures were adjusted by applying different processing parameters during the SLM fabrication. This approach allows modifying the mechanical properties within certain areas of the scaffold just by varying the process parameters during the fabrication process. Such gradients permit the fabrication of nature-analogue anisotropic structures. Furthermore, the pseudoelastic behavior of SLM-built NiTi specimens was demonstrated. Tailoring structural and related mechanical properties of NiTi-scaffolds, we aim to realize implants, which mechanically stimulate the tissue for an enhanced osseoinductive performance.

\section{Acknowledgements}

The multi-disciplinary team gratefully acknowledges the financial support of the Swiss National Science Foundation within the program NRP 62 'Smart Materials'.

\section{References}

[1] ASTM International, F 2063 - 05.

[2] T. Bormann, R. Schumacher, B. Müller and M. de Wild: Fabricating NiTi shape memory scaffolds by selective laser melting, eCells and Materials Journal, 22(Suppl.1): p. 12, 2011.

[3] H. Meier and C. Haberland: Experimental studies on selective laser melting of metallic parts, Materialwiss. Werkst., 39(9): p. 665-670, 2008.

[4] I. Yadroitsev, A. Gusarov, I. Yadroitsava, I. Smurov: Single track formation in selective laser melting of metal powders, J. Mater. Process. Technol., 210(12): p. 1624-1631, 2010. 